\title{
AERODYNAMIC MODELING OF OSCILLATING WING IN HYPERSONIC FLOW: A NUMERICAL STUDY
}

\author{
ZHU JIAN \\ China Academy of Aerospace Aerodynamics, \\ BeiJing, China \\ HOU YING-YU \\ Beijing Institute of Machine and Electron \\ BeiJing, China \\ JI CHEN \\ China Academy of Aerospace Aerodynamics, \\ BeiJing, China \\ LIU ZI-QIANG \\ China Academy of Aerospace Aerodynamics, \\ BeiJing, China \\ olion@sina.cn
}

Published 22 June 2016

\begin{abstract}
Various approximations to unsteady aerodynamics are examined for the unsteady aerodynamic force of a pitching thin double wedge airfoil in hypersonic flow. Results of piston theory, Van Dyke's second-order theory, Newtonian impact theory, and CFD method are compared in the same motion and Mach number effects. The results indicate that, for this thin double wedge airfoil, Newtonian impact theory is not suitable for these Mach number, while piston theory and Van Dyke's second-order theory are in good agreement with CFD method for $\mathrm{Ma}<7$.
\end{abstract}

Keywords: Rocket; piston theory; Van Dyke's second-order theory; Newtonian impact theory; unsteady aerodynamic force.

\section{Introduction}

Hypersonic aeroelasticity is an active area of research in nowadays. It is defined as the mutual interaction of inertial, elastic, and aerodynamic forces in a system, when there is feedback between deformation and flow. An important aspect of aeroelastic analysis within the hypersonic regime is the accurate of the unsteady aerodynamic forces.

Because of the limited capabilities of computational and experimental facilities, researchers have relied upon a number of approximate unsteady aerodynamic theories ${ }^{1-3}$ for the aeroelastic analysis of hypersonic vehicles. However, some experimental

This is an Open Access article published by World Scientific Publishing Company. It is distributed under the terms of the Creative Commons Attribution 3.0 (CC-BY) License. Further distribution of this work is permitted, provided the original work is properly cited. 
aeroelastic analysis of flutter boundary showed there was large difference between that predicted by approximate unsteady aerodynamic theories. Hanson ${ }^{4}$ observed At $\mathrm{Ma}=6.86$, the flutter boundary predicted using second-order piston theory aerodynamics was within $20 \%$ of the experimental flutter boundary. It also noted that the piston theory results were generally unconservative with respect to the experimental flutter boundaries. Goetz ${ }^{5}$ found, that between the theoretical and analytical results, of up to $20 \%$ were observed in the flutter velocity. In general, however, the combined Newtonian-piston theory approach generated conservative results, while the Netwonian approach was unconservative. Thus, an important issue is identification of modeling approaches for unsteady hypersonic aerodynamics that provide adequate fidelity in cases where classical approximate approaches become unreliable.

This work studies an oscillating double-wedge wing (Fig. 1) in hypersonic flow for different Mach numbers, using both approximate models and CFD solutions.

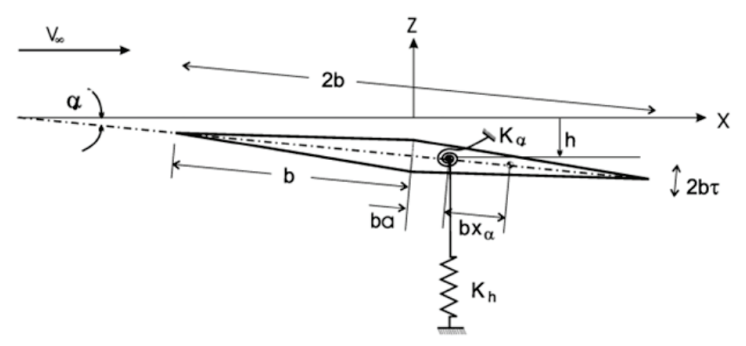

Fig. 1. A typical double-wedge wing.

\section{Approximate Aerodynamic Models}

The approximate models considered in this paper are briefly described in this section.

\subsection{Piston Theory}

Using this approximation of the flow, the position of a portion of solid wall, bounding a slab of fluid, moves normal to the flow with the velocity,

$$
w=\frac{d}{d t}(H(x, y, t)+\tau(x, y))
$$

where $H(x, y, t)$ is the position function of solid wall at time $t, \tau(x, y)$ is the thickness of wing at point $(x, y)$.

Assuming the piston generates only simple waves and no changes in entropy, the local pressure is therefore,

$$
\frac{P}{P_{\infty}}=\left(1+\frac{\gamma-1}{2} \frac{w}{a_{\infty}}\right)^{\frac{2 \gamma}{\gamma-1}}
$$

with ratio of specific heats $\gamma=1.4, P_{\infty}$ and $a_{\infty}$ are the pressure and sound speed of freestream, respectively. 
More popular formulas are second and third order binomial expansion of equation (2), the pressure coefficient on an oscillating surface is given by the following simple point function relationship:

$$
\begin{array}{r}
C_{p}(x, t)=\frac{2}{M_{\infty}^{2}}\left[c_{1} \frac{w(x, t)}{a_{\infty}}+c_{2}\left(\frac{w(x, t)}{a_{\infty}}\right)^{2}\right] \\
C_{p}(x, t)=\frac{2}{M_{\infty}^{2}}\left[c_{1} \frac{w(x, t)}{a_{\infty}}+c_{2}\left(\frac{w(x, t)}{a_{\infty}}\right)^{2}+c_{3}\left(\frac{w(x, t)}{a_{\infty}}\right)^{3}\right]
\end{array}
$$

where $M_{\infty}$ is the Mach number of freestream.

\subsection{Van Dyke's Second Order Theory}

Van Dyke's supersonic second order theory is an approximate aerodynamic theory commonly used in supersonic and hypersonic aeroelastic research. Its formula of the pressure coefficient is similar to second order Piston Theory,

$$
C_{p}(x, t)=\frac{2}{M_{\infty}^{2}}\left[\frac{M_{\infty}}{\beta} \frac{w(x, t)}{a_{\infty}}+\frac{M_{\infty}^{4}(\gamma+1)-4 \beta^{2}}{4 \beta^{4}}\left(\frac{w(x, t)}{a_{\infty}}\right)^{2}\right]
$$

with $\beta=\sqrt{M_{\infty}^{2}-1}$.

\subsection{Unsteady Newtonian Impact Theory}

Using this model, the change in momentum for a constant-area streamtube normal to the surface is given by,

$$
\left(\rho_{\infty} V_{\infty} \vec{n}\right) \cdot\left(V_{\infty}-V_{w}\right)=\left(P_{w}-P_{\infty}\right) \vec{n}
$$

where $\rho_{\infty}$ is the density of freestream, $\vec{n}$ is the normal vector to solid wall.

Since the normal component of momentum is zero at the body surface, this reduces to

$$
P_{w}-P_{\infty}=\rho_{\infty}\left(V_{\infty} \vec{n}\right)^{2}
$$

and finally the pressure coefficient is given by,

$$
C_{p}(x, t)=2 \sin ^{2}\left(\arctan \frac{w(x, t)}{V_{\infty}}\right)
$$

\section{Results and Conclusions}

Here, we assume that the angle of attack $\alpha$ of wing is changing as follow,

$$
\alpha(t)=\alpha_{0}+\delta \alpha \sin \left(\omega_{\alpha} t\right)
$$


where $\alpha_{0}$ is initial angle of attack, $\delta \alpha$ is the amplitude, the frequency is $\omega_{\alpha}=236.1 \mathrm{rad} / \mathrm{s}$.

For $\alpha_{0}=0, \delta \alpha=-\pi / 18, \mathrm{Ma}=5,6,7,8$. The lift coefficient is shown in Fig. 2.
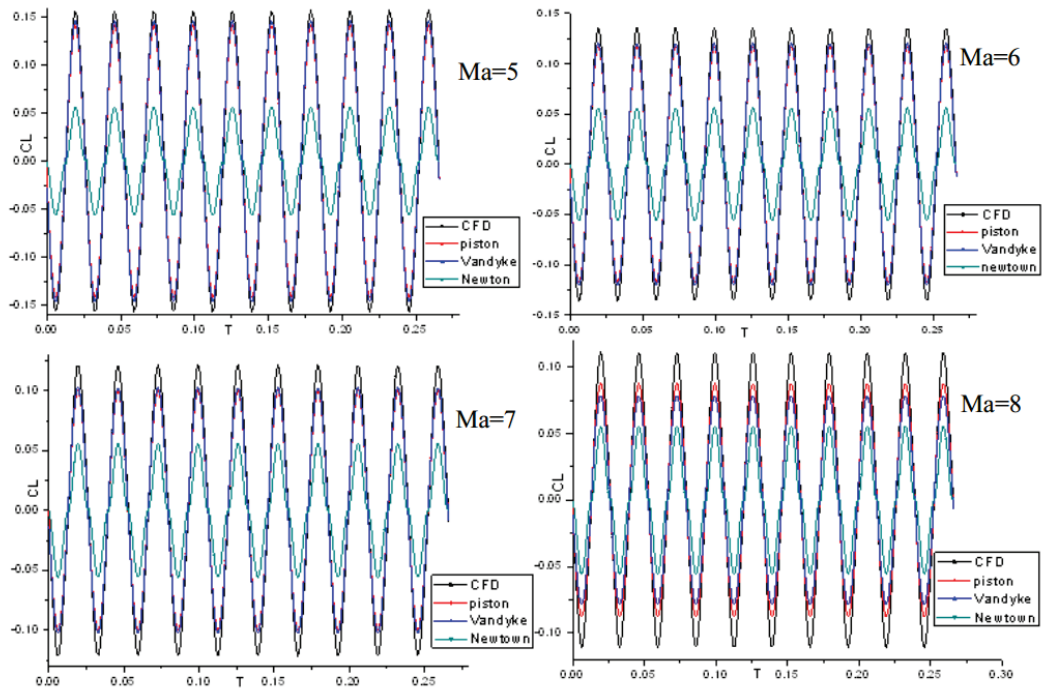

Fig. 2. Results of different Mach numbers.

For double-wedge wing and $\mathrm{Ma}<7$, the lift coefficients of piston theory and Van Dyke's second order theory are almost the same, the difference between them, as Mach number is increased, is about $5 \% \sim 12 \%$. The lift coefficients of Newtonian Impact Theory change slightly, its value is about $50 \%$ less than the CFD results. Comparing to CFD results, piston theory and Van Dyke's second order theory are closer. The results showed the Mach number effect of these method, for the case studied, piston theory and Van Dyke's second order theory maybe acceptable for $\mathrm{Ma}<7$, and Newtonian Impact Theory maybe acceptable for more large Mach number.

\section{References}

1. Lighthill, M. J., "Oscillating Airfoils at High Mach Numbers," Journal of the Aeronautical Sciences, 1953, 20, 402-406.

2. Ashley, H. and Zartarian, G., "Piston Theory-A New Aerodynamic Tool for the Aeroelastician," Journal of the Aeronautical Sciences, 1956, 23, 1109-1118.

3. Van Dyke, M., "A Study of Second-Order Supersonic Flow Theory,” 1951, NACATR 1081.

4. Hanson, P.W., "Aerodynamic Effects of Some Configuration Variables on the Aeroelastic Characteristics of Lifting Surfaces at Mach Numbers From 0.7 to 6.86," 1961, NASA TN D984.

5. Goetz, R.C., "Effects of Leading-Edge Bluntness on Flutter Characteristics of Some SquarePlanform Double-Wedge Airfoils at a Mach Number of 15.4," 1962, NASA TN D-1487. 